\title{
Resolution and validation of SAS-based structural models
}

Anne T. Tuukkanen ${ }^{1}$, Gerard J. Kleywegt ${ }^{2}$, Dmitri I. Svergun ${ }^{1}$

${ }^{1}$ European Molecular Biology Laboratory, Hamburg Unit, Hamburg, Germany, ${ }^{2}$ European Molecular Biology Laboratory, European Bioinformatics Institute (EMBL-EBI), Cambridge, United Kingdom

E-mail: annet@embl-hamburg.de

The possibility to reconstruct ab initio three-dimensional structures from one-dimensional small-angle-scattering (SAS) profiles of particles in solutions was a major breakthrough in the scattering techniques nearly two decades ago, and since then SAS has become a standard method for structural studies of macromolecules and their complexes. However, the models generated from SAS data are still reported without quantifying their resolution. This situation contrasts with other structural methods such as X-ray crystallography and electron microscopy. The lack of an objective quality measure for SASderived models has limited their validation and further use. Also, given the rapidly growing use of SAS in structural biology, it is of great importance to have common criteria for archiving of SAS data and models.

We have recently developed a method for resolution assessment of ab initio SAS models based on the average variability within a structural ensemble [1]. The average Fourier Shell Correlation (FSC) function over an ensemble of ab initio models was shown to reflect the variability of these models, which in turn was found to be directly related to the resolution of the individual models in the shape reconstruction. This approach was cross-validated using ab initio ensembles of proteins with known high-resolution structures with simulated and experimental SAXS data. Here, we shall demonstrate the practical use of the novel resolution measure and discuss its further extension for validation of SAS-based hybrid rigid body models. We expect the presented developments to have an impact on the archiving of SAS models and on their further use with other structural biology information.

[1] Tuukkanen AT, Kleywegt GJ, Svergun DI (2016). IUCr], 3, 440-447.

Keywords: small-angle scattering, ab initio models, hybrid rigid-body models 